\title{
Topological Schemas of Cognitive Maps and Spatial Learning
}

\author{
Andrey Babichev ${ }^{1,2}$, Sen Cheng $^{3}$ and Yuri A. Dabaghian ${ }^{1,2 *}$ \\ ' Department of Pediatrics Neurology, Baylor College of Medicine, Jan and Dan Duncan Neurological Research Institute, \\ Houston, TX, USA, ${ }^{2}$ Department of Computational and Applied Mathematics, Rice University, Houston, TX, USA, ${ }^{3}$ Mercator \\ Research Group "Structure of Memory" and Department of Psychology, Ruhr-University Bochum, Bochum, Germany
}

OPEN ACCESS

Edited by:

David Holcman

École Normale Supérieure, France

Reviewed by:

Tatyana Sharpee,

Salk Institute for Biological Studies,

USA

Dajiang Zhu,

University of Southern California, USA

${ }^{*}$ Correspondence:

Yuri A. Dabaghian dabaghia@bcm.edu

Received: 14 November 2015 Accepted: 16 February 2016

Published: 08 March 2016

Citation:

Babichev A, Cheng S and Dabaghian YA (2016) Topological Schemas of Cognitive Maps and Spatial Learning.

Front. Comput. Neurosci. 10:18. doi: 10.3389/fncom.2016.00018
Spatial navigation in mammals is based on building a mental representation of their environment-a cognitive map. However, both the nature of this cognitive map and its underpinning in neural structures and activity remains vague. A key difficulty is that these maps are collective, emergent phenomena that cannot be reduced to a simple combination of inputs provided by individual neurons. In this paper we suggest computational frameworks for integrating the spiking signals of individual cells into a spatial map, which we call schemas. We provide examples of four schemas defined by different types of topological relations that may be neurophysiologically encoded in the brain and demonstrate that each schema provides its own large-scale characteristics of the environment-the schema integrals. Moreover, we find that, in all cases, these integrals are learned at a rate which is faster than the rate of complete training of neural networks. Thus, the proposed schema framework differentiates between the cognitive aspect of spatial learning and the physiological aspect at the neural network level.

Keywords: hippocampus, learning and memory, topological analysis, mathematical concepts, theoretical neuroscience

\section{INTRODUCTION}

In the 1940's, Tolman proposed that animals build an internal representation-a cognitive mapof their environment and that this map allows the animal to perform space-dependent tasks such as navigating paths, finding shortcuts, and remembering the location of their nest or food source (Tolman, 1948). Three decades later, O'Keefe and Dostrovsky discovered pyramidal neurons in the hippocampus, named place cells, that become active only in a particular region of the environment-their respective place fields (Best et al., 2001) (Figure 1A). The striking spatial selectivity of these place cells led O'Keefe and Nadel (1978) to suggest that they form a neuronal basis of Tolman's cognitive map, thus providing this abstract concept with a concrete neurophysiological basis. In the ensuing decades, it was realized that there are many brain regions involved in cognitive mapping of the environment (Redish, 1997), yet there is still no consensus on either the physiological mechanisms of this phenomenon or the theoretical principles that explain them (McNaughton et al., 2006). Overall, it is believed that individual cells encode elements of the cognitive map, much like contributing pieces to a jigsaw puzzle. However, this analogy is not direct: the spiking activity of each separate neuron has no intrinsic spatial or geometrical properties-these properties appear only at the population level, emerging from the synchronous spiking activity of large neuronal ensembles (Eichenbaum, 1999; Pouget, 2000). The mechanism of this phenomenon remains unknown, i.e., there exists a disconnect between the level of individual neurons from which the preponderance of neurophysiological data is acquired and the level of neuronal ensembles 


\section{A}

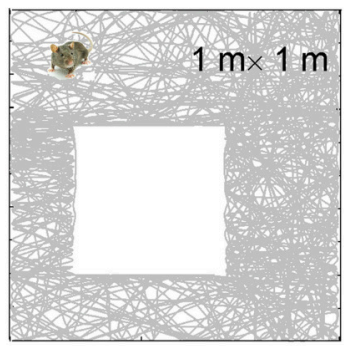

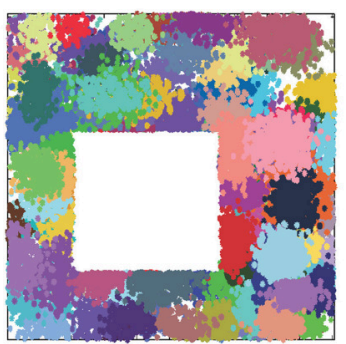

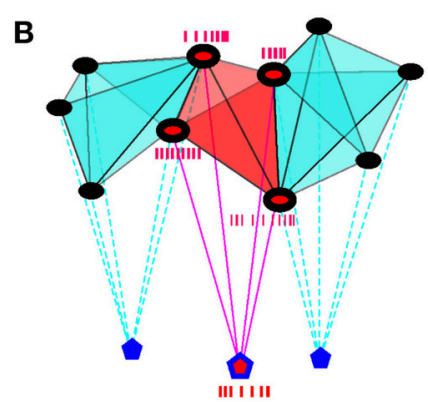

FIGURE 1 | Physiological components of the schema. (A) The simulated trajectory in a $1 \times 1$ m environment (left) and 200 randomly scattered place fields (clusters of colored dots) produced by place cells with a mean firing rate $f=12 \mathrm{~Hz}$ and a mean place field size $\mathrm{s}=20 \mathrm{~cm}$. (B) Schematic representation of three overlapping assemblies of place cells (shown by black dots) that project synaptically onto their respective readout neurons (blue pentagons). The active place cells (black dots with red centers) of the ignited cell assembly (in the middle of the figure) produce spike trains that drive the spiking activity of the readout neuron (blue pentagon with the red center).

where the large-scale representations of space are believed to emerge (Harnad, 1994).

In a recently proposed a model of spatial learning (Dabaghian et al., 2012; Arai, 2014), we attempted to bridge this gulf by combining recent experimental results pointing out the topological nature of the hippocampal map (Shapiro et al., 1997; Poucet and Herrmann, 2001; Alvernhe et al., 2011, 2012; Chen et al., 2012; Dabaghian et al., 2014; Wu and Foster, 2014) and methods of Algebraic Topology. This model allowed demonstrating that place cell activity can encode an accurate topological map of the environment and estimating the time needed to accumulate the required connectivity information. Further analyses of the model suggested to us that it is indicative of a more general theoretical framework that may lead to a systematic understanding of how spiking activity of neurons can be integrated to produce large-scale characteristics of space. In this paper, we outline the general principle and provide four specific models, which we call schemas, of integrating the activity of simulated neurons into a coherent representation of the explored environment. For each schema we find that a largescale spatial map is produced within a short, biologically plausible period, which could be used to estimate the spatial learning time in different environments.

\section{THEORETICAL FRAMEWORK}

\subsection{The Model}

A schema model of a cognitive map contains the following three key components:

\subsubsection{An Abstract Schema}

An abstract schema $\mathcal{S}\left(R, P_{S}\right)$ represents the spatial information contained in the map at any given time. It consists of a set of formal regions $R=\left\{r_{1}, r_{2}, \ldots, r_{N}\right\}$ and a set of relationships, $P_{S}=$ $\left\{\rho_{1}, \rho_{2}, \ldots, \rho_{M}\right\}$, that express how these regions combine. We presume that each region $r_{i}$ in the schema can be related to any other region $r_{j}$ through a chain of relationships with intermediate regions $\rho_{\alpha}\left(r_{i}, r_{k}\right), \rho_{\beta}\left(r_{k}, r_{l}, r_{m}\right), \ldots, \rho_{\gamma}\left(r_{n}, r_{j}\right)$. A specific selection of the relationships included in $P_{S}$ determines the type of spatial information encoded in the schema and the global arrangement of the encoded regions, which is crucial both for the properties of the resulting map as well as for the information encoded in it.

\subsubsection{The Neural Implementation}

The neural implementation, $\mathcal{N}_{S}$, is a neural network that encodes the schema $\mathcal{S}$. For the sake of simplicity, we model $\mathcal{N}_{S}$ using a basic, two layer, feed-forward neural network inspired by cell assembly theory (Buzsaki, 2010), which consists of a layer of cells that represent regions of space and another layer of readout neurons that represent the relationships between these regions (Figure 1B). When a cell $c_{i}$ fires a spike, we say that the region $r_{i}$ is "active"; otherwise it is "latent" (Russell, 1921). When a readout neuron fires a spike, we say that the corresponding relationship is "instantiated." Thus, by construction, the relationships between regions are represented via temporal relationships between the spike trains and by the parameters of synaptic connections between the two layers in $\mathcal{N}_{S}$.

\subsubsection{The Spatial Map and the Representing Space}

The goal of introducing schemas is to model the assemblage of the cognitive maps from the cells' spiking activity. However, in absence of a mechanism explaining how spatial representations emerge from the spike trains, this task remains undefined. Statements such as "a given place cell's activity encodes a region" or "the coactivity of a set of place cells represents a spatial overlap between the encoded regions" require an interpretation. In the analysis of electrophysiological data, this interpretation is acquired by mapping the neuronal activity into an auxiliary, external space $X$ which is selected according to the experimenters' best judgment. For example, constructing the place fields by ascribing Cartesian $x-y$ coordinates to the place cells' spikes and identifying the areas where the spikes cluster is one attempt to map the unobservable formal regions encoded in the cognitive map into observable regions of the spatial environment (Barbieri et al., 2004). In the following discussion, we will refer to this algorithm as to standard place field mapping. Spaces that have been used to interpret the activity of place cells and other cells include Euclidean domains in one (Frank et al., 2004; Diba 
and Buzsaki, 2008) and in three dimensions (Hayman et al., 2011; Yartsev and Ulanovsky, 2013); circles (Taube, 2011), tori (Finkelstein et al., 2015), spheres (Chen et al., 2011), and even Klein bottles (Swindale, 1996). To capture this aspect of cognitive map analysis, we define a spatial mapping from the schema $\mathcal{S}$ to a representing space $X$,

$$
f: \mathcal{S} \rightarrow X
$$

in which the formal regions of $\mathcal{S}$ are mapped into the "concrete" regions of $X, x_{k}=f\left(r_{k}\right)$. We will refer to $x_{k}$ as the $X$ representations of the formal region $r_{k}$ and to the resulting layout of the representing regions in $X$ as the spatial map of the schema, $M_{X}(\mathcal{S})$.

Although the representing regions are selected to reproduce the relationships between the formal regions as well as possible, the resulting map does not always capture the structure of the original schema: some relationships may be lost in the mapping or the mapping may produce relationships between the representing regions that are not encoded in $\mathcal{S}$. For example, the place field maps are believed to reflect an animal's cognitive map's structure but their faithfulness has not been established or even addressed in the neurophysiological literature. In the case when the set of relationships between the regions $x_{k}\left(P_{X}\right)$ matches the schematic relationships exactly, so that $P_{X}=P_{S}$, the mapping will be referred to as faithful. The corresponding spatial map may then be viewed as a model of $\mathcal{S}$, i.e., the structure of $\mathcal{S}$ can be deduced from the layout of the representing regions.

Thus, each specific schema model includes these three components-the abstract schema $\mathcal{S}$, its neuronal network implementation $\mathcal{N}_{S}$ and the spatial mapping (1) into a representing space $X$. For brevity, we will refer to this triad as to "schema," when no ambiguities can arise.

\subsection{Spatial Learning}

A key property of our approach, crucial for modeling spatial learning, is that schemas are dynamic objects. As an animal explores a novel environment, new regions become represented by the activity of place cells and new relationships are inferred from the spike trains' temporal patterns (Dabaghian et al., 2012; Arai, 2014). According to the standard approach of neural network theory, the process of learning a schema may be viewed as the process of training the readout neurons to represent the set $P_{S}$ by detecting repetitive patterns in the incoming spike trains. From this perspective, a schema is learned after its network is trained, i.e., after the readout neurons stop adopting their spiking responses to the patterns of the incoming spike trains.

On the other hand, from a cognitive perspective, the purpose of spatial learning is to acquire qualitative, largescale characteristics of the environment, which enable spatial planning, spatial navigation and spatial reasoning, such as path connectivity, shortcuts and obstacles, geometric and topological properties, global symmetries and so forth. Such large-scale characteristics of the environment that are captured through the relationships of a given schema will be referred to as schema integrals, $I_{\mathcal{S}}$. Below we demonstrate that the minimal time $T_{\min }$ required for the schema's integrals to emerge is typically shorter than the time, $T_{\mathcal{N}}$, required to train all readout neurons, i.e., large-scale information can be extracted from a partially trained network. Thus, the schema approach captures two complementary aspects of spatial learning: physiological learning - the process of forming and training the cell assembly network and schematic or cognitive learning - the emergence of information about the global structure of space, expressed as the corresponding production of schema integrals.

\subsection{Topological Schemas}

What aspect of space is represented in the hippocampal map? The answer to this question depends on the information captured by the readout neurons in the hippocampal cell assemblies. Since correlating neuronal spiking with geometrical properties of the representing space sometimes produces useful interpretations of electrophysiological data, most authors assume that the spiking patterns of place cells encode geometric properties of space (O'Keefe and Burgess, 1996; Barry and Burgess, 2007). For example, it has been shown that combining the spiking activity of a relatively small number of place cells with the information about the sizes, shapes, and locations of their respective place fields allows a reconstruction of the animal's trajectory in a typical experimental enclosure on a moment-to-moment basis (Brown et al., 1998; Guger et al., 2011).

However, the read-out neurons have access only to the place cells' spikes, and not to their respective place fields. Obtaining the shape and size of any given place field, which is nothing but a cumulative spatial histogram of spikes used for illustrational purposes, requires accumulating a substantial number of spikes from the corresponding place cell. Yet the spike trains produced during the activity period of a given place cell are shorttypically hundreds of milliseconds in duration-and highly variable, not only because of the animal's movements, but also because of the intrinsic stochasticity of neuronal spiking (Fenton and Muller, 1998). Thus, the spike trains of place cells contain little information about a place field, such as its shape, location and other computationally expensive parameters. Furthermore, recent experimental studies point out that these spike trains do not provide the geometric information on the synaptic integration timescale of seconds or fractions of a second (Diba and Buzsaki, 2008; Cheng and Ji, 2013; Dabaghian et al., 2014).

Since the temporal pattern of place cell firing is the only information available to downstream neurons, a physiologically adequate class of schemas of the hippocampal map may be constructed based on capturing qualitative, topological relationships between regions, e.g., overlap, adjacency, ordering and containment, from the temporal relationships between the spike trains (Dabaghian et al., 2011; Chen et al., 2012). The resulting maps will then produce a topological representation of space rather than a geometrical one (Stella et al., 2013; Chen et al., 2014), in which the relative arrangement of the locations is more important than mapping the precise positions. Topological schemas have several advantages over the more precise geometric schemas, e.g., higher stability (e.g., faithfulness of a topological map is not destroyed under continuous deformations of the representing space) and lower computational cost, which may make them biologically more viable (see Section 4). 
There remain many possibilities in which to read out qualitative information about the spike trains and thus there are many topological schemas. In this perspective, a particular readout mechanism, which responds to specific patterns of place cell coactivity, defines the type of spatial information encoded in the schema. The following discussion presents four different topological schemas based on different qualitative relations between regions and the rate at which these schemas are acquired.

\section{RESULTS}

\subsection{Graph Schema $\mathcal{G}$}

The simplest topological schema is based on binary connections: its set of relationships consists of pairs of connected regions, $P_{G}=\left\{\left(r_{i}, r_{j}\right),\left(r_{i}, r_{k}\right),\left(r_{m}, r_{n}\right), \ldots\right\}$. Such schema can be viewed as a graph, $G$, whose vertices are linked if the corresponding regions are related according to $P_{G}$ (Figure 2). The corresponding neuronal implementation is produced by training the paircoactivity detector readout neurons to respond to nearly simultaneous spiking of their respective pair of presynaptic cells (Katz et al., 2007; Brette, 2012). In other words, physiological learning of a graph schema $\mathcal{G}$ amounts to detecting pairs of cells that exhibit frequent coactivity (Muller et al., 1996).

We modeled this process by simulating place cell spiking activity induced by a rat's movements across a place field map in a small environment (Figure 1B). To simplify the analyses, we assume that as soon as the coactivity occurs, the corresponding connection is immediately "learned," i.e., incorporated into the schema. As a result, at every moment of time $t$, the connectivity matrix of the graph is defined by the coactivity observed prior to that moment. Thus, $C_{i j}=1$ if cells $c_{i}$ and $c_{j}$ cofired before $\mathrm{t}$ and $C_{i j}=0$ otherwise. Figure $\mathbf{3 A}$ shows that the number of links in the graph, which is the number of recruited pair-coincidence detectors, grows as the schema is learned and saturates at ca. $T_{\mathcal{N}}=5$ mins, i.e., after this time new incoming spike trains do not produce new connections in $\mathcal{N}_{G}$.

The saturation of the schema could be a trivial result if the graph becomes fully connected or remains mostly empty. A simple characteristic capturing the efficiency of $\mathcal{G}$, which generalizes to other schemas in a natural way is its entropy
(Mowshowitz, 1968; Dehmer and Mowshowitz, 2011). This is the specific entropy of the readout neurons,

$$
H_{G}=-p_{c} \log _{2}\left(p_{c}\right)-p_{d} \log _{2}\left(p_{d}\right)
$$

where $p_{c}$ and $p_{d}=1-p_{c}$ are the fractions of the connected and disconnected vertex pairs in the graph. For a fully discrete $\left(p_{c}=0\right)$ or a fully connected graph $\left(p_{c}=1\right)$ the entropy vanishes and maximal entropy $H_{G}=1$ is achieved for $p_{c}=1 / 2$ (in which case the absence of a link is as informative as its presence). Figure 3B demonstrates that for the place cell ensemble used in our simulations (see below, see Methods), the entropy of the graph schema asymptotically approaches a maximal value of about $H_{G} \approx 0.8$ in about $5 \mathrm{~min}$, a value implying that the schema network $\mathcal{N}_{G}$ is neither underloaded nor oversaturated.

To quantify the correspondence between the schema $\mathcal{G}$ and its place field map $M_{X}(\mathcal{G})$, we calculated the entropy $H_{X}$, of the occurrences of place field pairwise overlaps across time and compared $H_{X}$ to $H_{G}$. Figure $3 \mathbf{B}$ demonstrates that both entropies remain close throughout the entire learning period, indicating that the complexity of the place field layout remains similar to the complexity of the encoded relationships at all times. In addition to this correspondence we also computed the mutual information (MI, see Methods) between the place field overlap and place cell coactivities, which also grows with the rat's navigational experience (Figure 3B). Thus, we have convergent lines of evidence indicating efficient spatial learning captured by the graph schema.

As a cognitive map model, the graph schema $\mathcal{G}$ provides a stratum for implementing graph-theoretical navigation algorithms, that is, for establishing paths connecting spatial locations (Trullier and Meyer, 2000; Chrastil and Warren, 2014). Its integrals $I_{\mathcal{G}}$ are the global characteristics of the region-to-region connectivity graph, e.g., its partitioning, the colorability of its vertexes and edges (Berge, 1982), its planarity, and the existence of a path between two given vertexes. As an example of such large-scale characteristics we identified the shortest paths connecting pairs of the most distant vertexes and computed the time required to establish these connections. The results shown in Figure $\mathbf{3 C}$ demonstrate that the animal
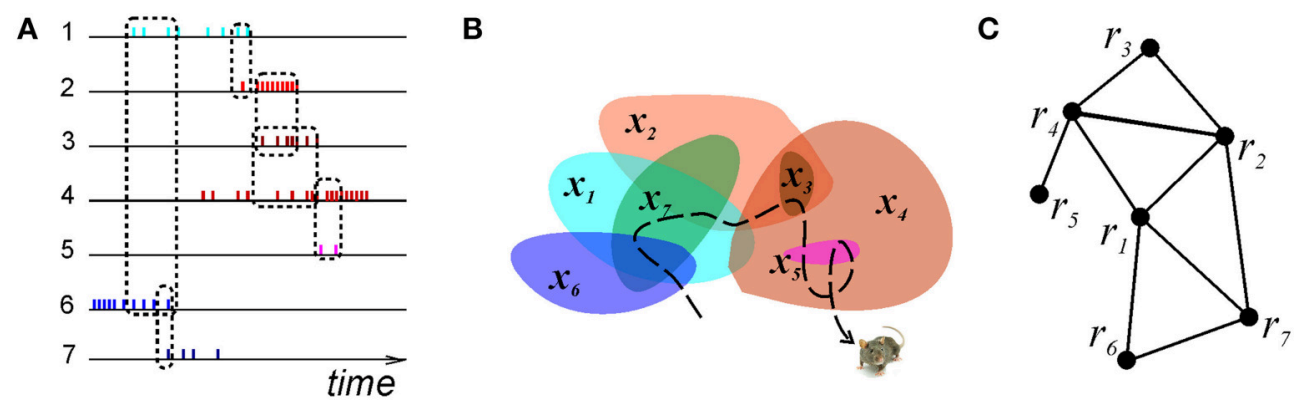

FIGURE 2 | Graph schema. (A) A schematic illustration of the spike trains produced by seven place cells whose coactivity is indicated by the dashed rectangles connecting the spike trains. (B) The corresponding seven place fields traversed by the animal's trajectory (dashed line). (C) The corresponding graph schema, the seven vertexes of which correspond to seven formal regions encoded by the active place cells. The edges mark the relationships encoded in the schema, e.g., the connection $\left(r_{4}, r_{5}\right)$ is in the schema, but $\left(r_{5}, r_{1}\right)$ is not. 

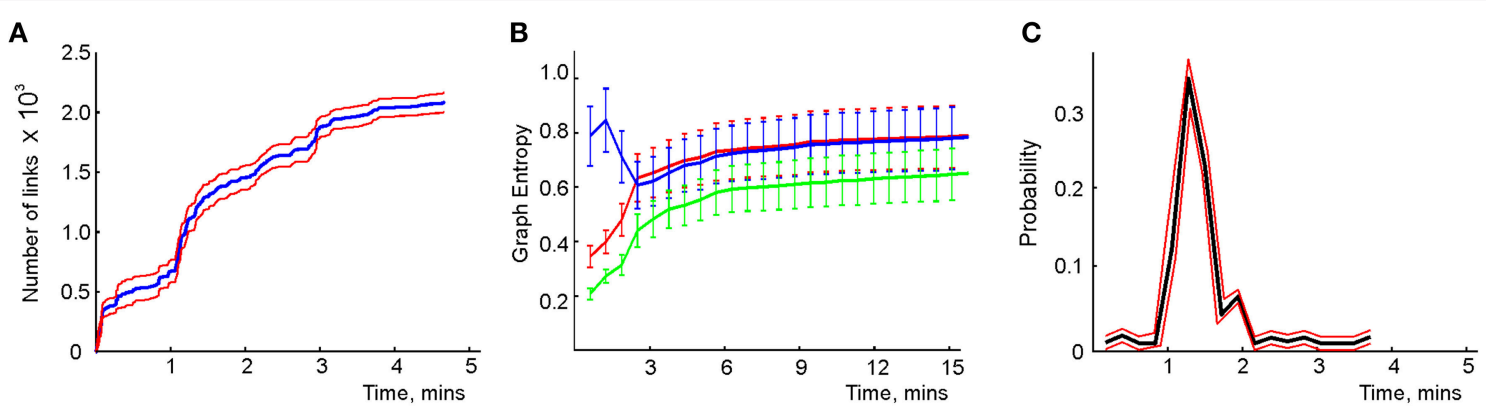

FIGURE 3 | Spatial learning based on the graph schema. (A) The number of links in the graph schema as a function of time, computed for a simulated ensemble of 200 place cells with randomly scattered place fields (see Section 5). The blue line represents the mean and the red lines show the error margins. (B) Graph schema entropy (blue) and place field map entropy (red) as a function of time. The green line shows the mutual information between the map and the schema. Both entropies and the mutual information saturate at the time when the number of links saturates. (C) The probability of establishing a maximal length connection in the graph schema stabilizes in about 2.2 min, when only $50 \%$ of connections appeared.

establishes connections between the most distant locations in the graph in about $T_{\min }=2.2 \mathrm{~min}$, a time when only about $50 \%$ of the readout neurons are trained. Similarly, emergence of the information required to establish existence of a circuit of the graph which traverses each edge exactly once, called an Eulerian path, takes about $2.2 \mathrm{~min}$, while the correct number of cliques in $\mathcal{G}$, which are sets of pairwise connected vertices, can be deduced within $2 \mathrm{~min}$. These observations suggest that the emergence of schema integrals before the network is trained may be a general phenomenon.

\subsection{Higher-Order Overlap (Simplicial) Schema $\mathcal{T}$}

A topological schema may be based on representing not only binary, but also ternary, quaternary and other higher-order connectivity relations between spatial domains. For example, a schema may represent the overlaps between regions, including triple, quadruple, etc., overlaps. The key property of the overlap relation is that if $k$ regions, $r_{1}, r_{2}, \ldots, r_{k}$, have a common intersection, then so does any subcollection of them. The simplest mathematical object that is closed under the operation of taking non-empty subsets is an abstract simplex, which can be viewed as a list of $k$ elements (Aleksandrov, 1965). Hence, a $(k+1)$ order overlap relationship $\rho\left(r_{0}, r_{1}, \ldots, r_{k}\right)$ may be represented by a $k$-dimensional simplex $\sigma=\left[r_{1}, r_{2}, \ldots, r_{k}\right]$. A set of overlap relationships therefore forms an abstract simplicial complex, $\mathcal{T}$, and we will hence refer to a higher order overlap schema as to simplicial schema.

Under the standard mapping of the place cell spiking activity into the environment, the simplicial schemas' relationships, $P_{\mathcal{T}}$, represent the overlaps between the place fields. For example, the place field map shown in Figure $\mathbf{2 B}$ can be faithfully encoded by a simplicial schema with four $3^{d}$ order relationships $P_{3}=\left\{\left(r_{6}, r_{1}, r_{7}\right),\left(r_{7}, r_{1}, r_{2}\right),\left(r_{1}, r_{2}, r_{4}\right),\left(r_{2}, r_{3}, r_{4}\right)\right\}$ and an additional binary relation $\left(r_{4}, r_{5}\right)$, as shown in Figure 4. The neuronal marker of these overlaps is the spiking coactivity: if the animal enters a location where several place fields overlap, their respective place cells produce (with a certain probability) temporally overlapping spike trains. Hence the neural network implementation of a simplicial schema, $\mathcal{N}_{\mathcal{T}}$, should be built to detect the coactivity events, using coincidence detector readout neurons [which, in fact, corresponds to the current view on the hippocampal cell assembly network organization (Harris et al., 2003; Harris, 2005; Buzsaki, 2010; Babichev et al., 2015)].

Physiological learning of a simplicial schema hence amounts to training the readout neurons to detect place cell coactivities. Our learning algorithm (see Section 5) ensures that, at every stage of learning, only the highest order relationships are kept while the redundant lower-order relationships are eliminated. For example, pairwise connections between three neurons become redundant after a triple coactivity between them is detected, at which point the three pair-detector readout neurons can be replaced with a single triple-coincidence detector. Numerical simulations demonstrate that, as the rat explores the environment, the more probable, lower-order coactivity events are captured first and the less probable higher order coactivities accumulate more slowly (Figure 5A). Moreover, although rapid changes of the readout neurons' order stops after 5 or $6 \mathrm{~min}$, slow regroupings continue during the entire navigation period, $T=25$ min. Thus, unlike the pairwise overlaps in $\mathcal{G}$ that can be instantly identified, the orders of the readout neurons cannot be deduced from a single coactivity event. In this sense, the orders of the readout neurons are integral characteristics of place cells' spiking activity, and therefore may be viewed as integrals of the simplicial schema.

There exists an additional important set of $\mathcal{T}$-integrals, which capture the topology of the representing space. This property of the simplicial schemas can be illustrated using the Čech theorem, which states that the pattern of overlaps between regions $U_{1}$, $U_{2}, \ldots, U_{n}$, covering a topological space $X=\cup_{i} U_{i}$, encodes homological invariants of $X$, provided that every intersection $U_{i} \cap U_{j} \cap \ldots \cap U_{k}$ is contractible (Dubrovin et al., 1992; Hatcher, 2002). The proof is based on building the "nerve" of the covering - a simplicial complex, the $d$-dimensional simplexes of which correspond to the $(d+1)$-fold overlaps between covering regions, and showing that it is topologically equivalent to $X$ (Figure 4C). This theorem implies that the spatial map of a sufficiently rich simplicial schema may encode the topology of 
A

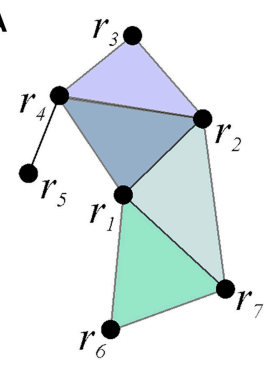

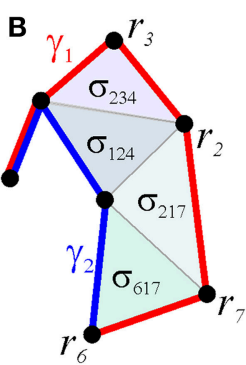

C

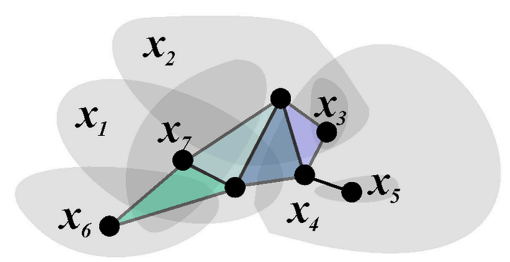

FIGURE 4 | Simplicial schema. (A) schematic representation of the connections between the vertexes associated with the seven place cells and their spatial map shown on Figure 1B. (B) The existence of the two-dimensional simplexes, corresponding to higher order coactivity relationships, permits the links to be deformed. This is illustrated for paths $\gamma_{1}$ and $\gamma_{2}$ : the transformation can be visualized by slipping one path across the two-dimensional facets, thus demonstrating the topological equivalence between paths $\gamma_{1}$ and $\gamma_{2}$. (C) The nerve of the map shown on Figure 1B matches the simplicial schema.
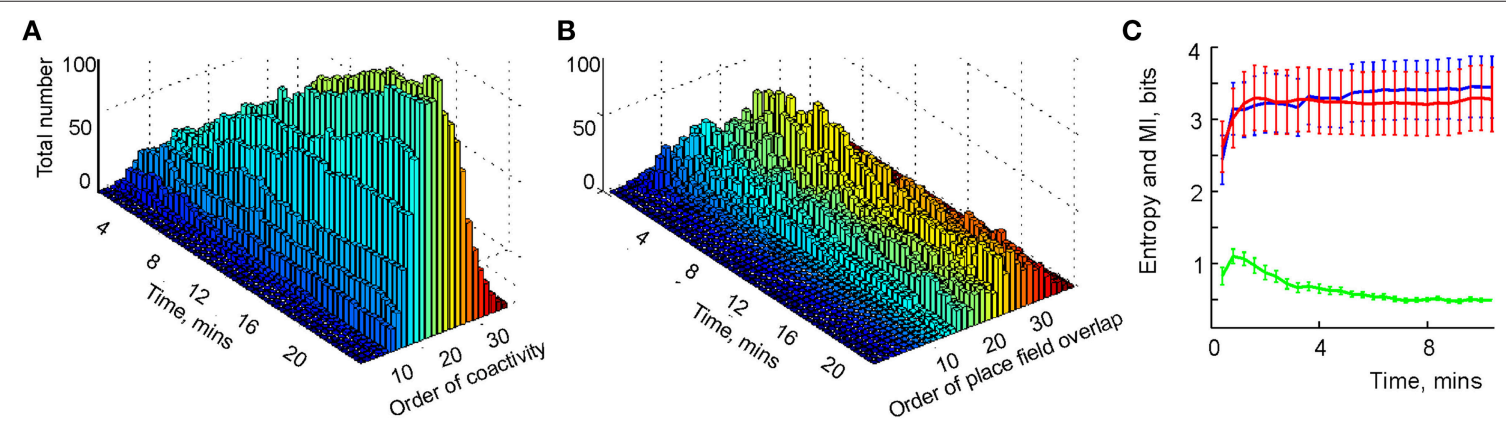

FIGURE 5 | Learning the simplicial schema. (A) The development of the population of readout neurons as a function of time. The typical order of co-activity is about 20, the highest order is 33 . The population of the high order readout neurons (order above 11) increases through the entire duration of the experiment (over 20 min). Other populations reach a stable plateau (e.g., orders 9 and 10) or reach a maximum and then drop (e.g., order under 8). The decrease of the number of the low order relationships after an initial increase indicates the elimination of redundant information. (B) The development of the overlap relationships between the standard representing regions as a function of time. The typical overlap order is about 20 , the highest order is 35 . As in the case with the readout neurons, the number of high-order overlaps (order above 17) saturates on the rise, unlike the lower order overlaps. (C) The entropy of the dimensions of registered simplexes (red) is similar to the entropy of the orders of the overlaps of the concrete regions $x_{k}$ (blue). The mutual information between these two variables is computed along the trajectory (green).

the space navigated by the rat, and suggests that if this map is faithful, i.e., if the nerve of the spatial map matches the schema's relationship set $P_{\mathcal{T}}$ exactly, then the schema also captures the large-scale topological representation of the space.

To study the correspondence between the simplicial schema and its map, we compared the schema's entropy $H_{\mathcal{T}}$, defined by the probabilities for a readout neuron to become a $k^{\text {th }}$ order co-activity detector, to the entropy $H_{X}$ of the place field map $M_{X}(\mathcal{T})$, defined via the probabilities of producing a $k^{\text {th }}$ order overlap between the place fields (Figure 5B). As shown on Figure 5C, both entropies closely follow one another: they both grow initially and reach similar asymptotes in approximately 4 min. However, the mutual information between these two series of events decreases with time. The reason for this effect lies in the idealized nature of the representing regions $x_{k}$, built as convex hulls of the spike clusters in the two-dimensional environment (for other place field construction algorithms see Muller et al., 1987; Maurer et al., 2006). The $x_{k}$ 's crisp boundaries produce high-order overlaps, which are not captured by the place cell coactivity and hence by the schema-compare the orders of the readout neurons on Figure 5A with the orders of overlap between the corresponding representing regions $x_{k}$ in Figure 5B.
This result can be viewed from several perspectives. First, it illustrates that the scope of reliable information that can be drawn from the spatial map is limited: only sufficiently robust, qualitative aspects of the place field map, such as low dimensional overlaps, can be trusted. Second, the regions $r_{i}$ that were originally introduced as "formal," that is, devoid of intrinsic properties, should fundamentally be viewed as "fuzzy" and not as Euclidean domains with crisp boundaries (Liu and Luo, 1997).

Direct computations show that the coactivity complexes do, in fact, capture the topology of the representing space, provided that the place cells' spiking parameters fall into the biological domain (Curto and Itskov, 2008; Dabaghian et al., 2012; Arai, 2014; Babichev et al., 2015), and hence that simplicial schemas provide a framework for representing topological information. For example, cell assemblies ignited along the physical paths traversed by the animal correspond to sequences of coactivity simplexes-the simplicial paths that represent the physical paths in $\mathcal{T}$ (Figure 6A). The structure of the simplicial paths allows establishing topological (in)equivalences between navigational paths, e.g., topologically equivalent simplicial paths represent physical paths that can be deformed into one another, a non-contractible simplicial 
A

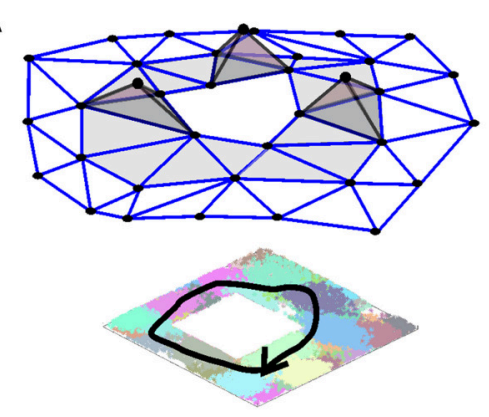

B

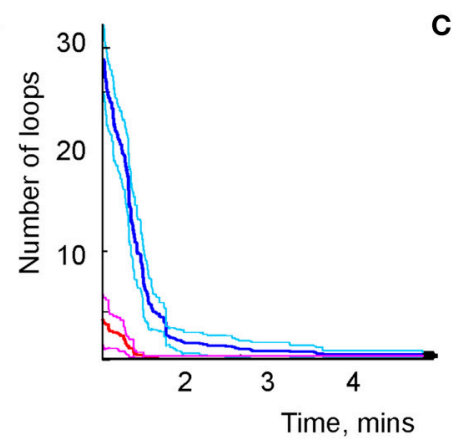

C

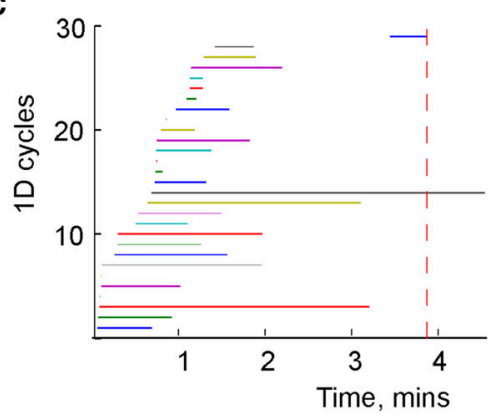

FIGURE 6 | Topological loops in simplicial schema. (A) Sequence of the place cell combinations ignited along a path $\gamma$ (black line) corresponds to a sequence of simplexes - a simplicial path $\Gamma$ that represents $\gamma$ in $\mathcal{T}$. (B) The dynamics of the total number of zero-dimensional loops (red) and one-dimensional loops (blue). Unlike the growing number of links in the graph schema (Figure $\mathbf{2 A}$ ), the number of topological loops decreases with time. Eventually, a single loop survives. The margins of error are shown above and below each graph by a pair of pink and light-blue lines, respectively. (C) The barcodes - timelines of the one-dimensional loops in the simplicial complex. The topological noise vanishes after ca. $4 \mathrm{~min}$, which is the schematic estimate of the cognitive learning time.

path corresponds to a class of the physical paths that enclose inaccessible or yet unexplored parts of the environment. As a result, the simplicial schema produces a qualitative description of navigational routes: while the total number of paths grows exponentially, the number of topologically distinct loops, which represent topologically distinct paths is small (Figure 6B).

However, this information does not emerge immediately: as the animal begins to navigate a new environment, most topological loops reflect transient connections. As the spiking information accumulates, these "spurious" loops disappear and only the loops that correspond to the physical signatures of the environment persist (Figure 6C). With methods drawn from persistent homology theory (Zomorodian and Carlsson, 2005; Ghrist, 2008) one can determine the minimal period $T_{\min }$ required for removing the spurious loops, which provides a theoretical estimate of the time required to learn the environment (Dabaghian et al., 2012; Arai, 2014). Figure 6C demonstrates that in our test map, after $T_{\min }=4 \mathrm{~min}$ most topological loops have vanished and only the loops that correspond to the physical holes in the environment survive. Thus, as in the case of the graph schema, the topological connectivity of the cognitive map is captured by the simplicial schema before the underlying neuronal network is fully trained, $T_{\min }<$ $T_{\mathcal{N}}$

\subsection{Mereological Schema $\mathcal{M}$}

Although a sufficiently rich simplicial schema can capture the topological invariants of the representing space $X$ as its integrals, it does not capture all the qualitative topological aspects of the connectivity between regions. For example, the identical simplicial schema (represented by a tetrahedron) can faithfully represent the overlap relationships in the two maps shown in Figure 7, because both maps contain the same set of regions $R=\left\{r_{1}, r_{2}, r_{3}, r_{4}\right\}$ and one fourth-order overlap relation $P_{R}=\left\{\left(r_{1}, r_{2}, r_{3}, r_{4}\right)\right\}$, as well as all their consequent ternary and binary sub-relations. However, these maps are topologically different, since they cannot be transformed from one into another by a continuous deformation of the plane $R^{2}$. The obstruction to such deformation is that the region $x_{4}$ on Figure $7 \mathrm{~A}$ is contained in the union of the regions $x_{1}$, $x_{2}$ and $x_{3}$, i.e., $x_{4} \subset\left(x_{1} \cup x_{2} \cup x_{3}\right)$, and no containment relationships exist between any combinations of the regions on Figure 7B. Neither a graph schema $\mathcal{G}$ nor a simplicial schema $\mathcal{T}$ can capture this difference; what is required is the additional covering relation, $\left(x_{1}, x_{2}, \ldots, x_{l}\right) \triangleleft\left(y_{1}, y_{2}, \ldots, y_{k}\right),\left(x^{\prime} s\right.$ are covered by $y^{\prime}$ s), in terms of which the map on Figure 7A is described by the relationship $r_{4} \backslash\left(r_{1}, r_{2}, r_{3}\right)$, whereas the regions shown on Figure $\mathbf{7 B}$ produce no containment relation. The cover relation produces a new-mereological-schema $\mathcal{M}$, in which the information is encoded in terms of topological containment (Figure 7C). The intuition behind neuronal implementation of the formal cover relation is the following. If the activity of one ensemble of place cells, $U=\left\{c_{1}, c_{2}, \ldots, c_{k}\right\}$, outlasts, or covers in time, the activity of cells in another ensemble $V=\left\{d_{1}, d_{2}, \ldots, d_{l}\right\}$, then the region $X_{U}$, representing the $U$-ensemble, contains the region $X_{V}$ representing the $V$-ensemble:

$$
X_{V} \subset X_{U} \text { if } V \triangleleft U .
$$

From this perspective, the set of covering cells provides contextual information about the covered cells, i.e., the cover relation combines the basic formal regions into more complex, composite regions.

The cover relationship can be implemented, e.g., by a combination of the excitatory and inhibitory neurons shown on Figure 7D. In such a cell assembly, the readout neuron signals a violation of the cover relationship, i.e., the latter is represented by an absence of the readout neurons' spiking activity up to the moment $t$. Hence, in contrast with simplicial schemas, where readout neurons learn to detect ever higher-order coactivities, a readout neuron in a mereological schema $\mathcal{M}$ learns to detect ever larger groups of cells that together inhibit its activity (Figure 7D).

Similarly to the overlap orders in $\mathcal{T}$, the cover relationships, as a rule, cannot be deduced from a single coactivity event. Thus, these relationships represent integral information that 

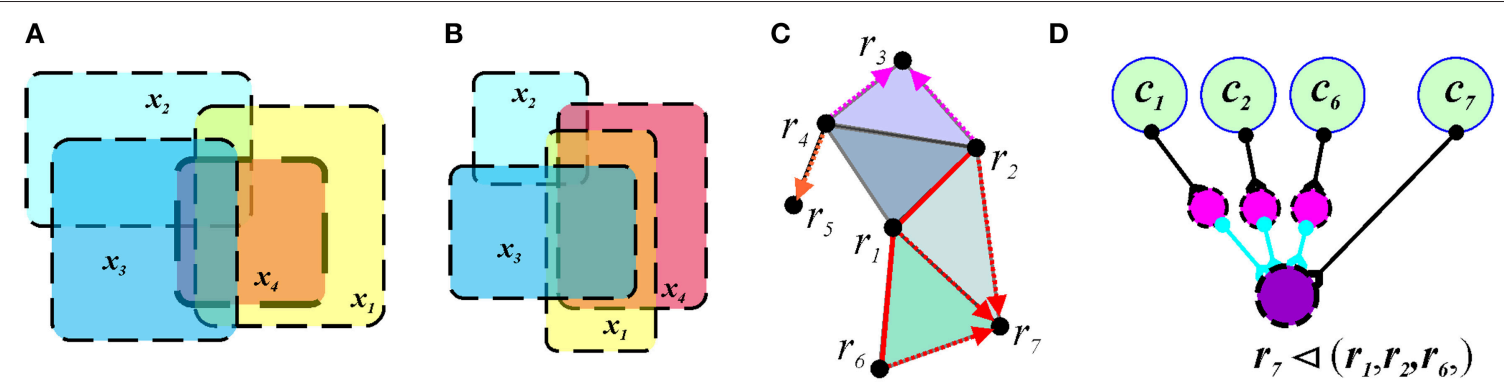

FIGURE 7 | Mereological schema. In panels (A,B), the overlap pattern does not capture the cover relationship. Four regions, $x_{1}, x_{2}$, $x_{3}$, and $x_{4}$ form a quadruple overlap in both cases. However, in map shown in (A), the region $x_{4}$ is contained in the union of $x_{1}, x_{2}$, and $x_{3}$. In the map shown in (B), $x_{4}$ is not covered. (C) The cover and the overlap relationships in a mereological schema corresponding to the map of Figure $\mathbf{1} \mathbf{A}$. The covering regions are connected by red links (e.g., $r_{6}, r_{1}$, and $r_{2}$ ) and the red arrows point to the covered region (e.g., $r_{7}$ ). (D) A neuronal implementation of the covering relationship includes three inhibitory neurons (magenta) that provide inhibitory input into the readout neuron (purple). If each inhibitory input of an active interneuron exceeds the excitatory input of the driving cell $c_{7}$, the readout neuron can spike only if the activity of the cell $c_{7}$ is not accompanied by the inputs from any of the cells $c_{1}, c_{2}$, or $c_{6}$. As a result, the readout neuron will remain silent as long as the activity of the cells $c_{1}, c_{2}$, and $c_{6}$ temporally covers the activity of cell $c_{7}$.

can be viewed as the $\mathcal{M}$-integrals which characterize the large-scale topology of a space. We are currently unaware of additional mereological algorithms that would allow largescale computations of the environment's global topological characteristics, similar to computing the homological invariants in a simplicial schema. Nevertheless, a mereological schema encodes an important type of topological information, which may be used in physiological neural networks to represent spatial maps.

In general, covering relationships can be established between arbitrary (including multiply connected) regions. As a result, the number of possible combinations of covered and covering regions dramatically increases. Even if the covered region $V=$ $\left\{d_{1}, d_{2}, \ldots, d_{l}\right\}$ is spatially "bundled" (e.g., if each pair $d_{i}$ and $d_{j}$ is coactive at some moment of time, so that $V$ forms a connectivity clique) the selection of possible covering regions remains very large. Therefore, in order to test the development of cover relationships in time, we opted to limit our study to neuron pairs covering an individual neuron $(k=2$ and $l=1)$.

The results of simulations show that the time required to learn second-order covering relationships in $\mathcal{M}$ is comparable to the learning times in the graph schema $\mathcal{G}$ (Figure 8). As spatial exploration begins, a large number of transient covering relationships is produced because of insufficient spiking data. With accumulating spike trains most cover relationships become violated, so that the number of surviving relationships quickly drops. As the animal completes its first turn around a central hole of the environment (Figure 1B), a new set of (mostly transient) relationships is injected into the schema which produces the peak shown in Figure 8B. Subsequently, the number of cover relations steadily diminishes to about 200 pairs, which corresponds to a saturated schema. This result reflects qualitative behavior of higher order covering relationships, though a full implementation of the algorithm for the higher-order covering combinations $(k, l>1)$ is computationally substantially more complex.

\subsection{Complex Relations and the RCC Schema $\mathcal{R}$}

Qualitative Space Representations (QSR) are discrete, regionbased versions of the conventional point-set theoretical geometries and topologies (Hazarika and Cohn, 2001) used to formalize "intuitive" qualitative spatial reasoning (Gotts et al., 1996; Cohn et al., 1997; Renz, 2002), and thus are particularly important for modeling cognitive representations of space (Knauff et al., 1997; Goodrich-Hunsaker et al., 2008; Wallgrn, 2010; Zeithamova et al., 2012). Important examples of QSRs are the Region Connection Calculi (RCC)_formal logical theories based on a family of binary topological relations between regions (Cui et al., 1993). For example, the most basic RCC theory, RCC5, which applies to the case of regions with fuzzy boundaries, is built using the five relations shown in Figure 9A: disconnect (DR), partial overlap (PO), proper part and its inverse (PP and PPi), and equality (EQ) (Cohn et al., 1997). In terms of these relations, the arrangement of regions shown on Figure 2B is described by the following set of RCC5 relationships: $P=\left\{\mathrm{PO}_{12}, \mathrm{PO}_{14}, \mathrm{PO}_{16}, \mathrm{PO}_{17}, \mathrm{PPi}_{23}, \mathrm{PO}_{24}, \mathrm{PO}_{27}, \mathrm{PP}_{32}, \mathrm{PP}_{34}\right.$, $\mathrm{PPi}_{43}, \mathrm{PPi}_{45}, \mathrm{PO}_{46}, \mathrm{PP}_{54}, \mathrm{PO}_{67}$; DR for all other pairs (Figure 9B). More elaborate RCC calculi can capture tangencies (Cui et al., 1993), convexity (Cohn et al., 1997), qualitative directions ( $\mathrm{Li}$ and Cohn, 2012), and distances (Gerevini and Renz, 1998) as well as complex hierarchies of all these relationships (Lehmann and Cohn, 1994). As a result, RCC methods can capture not only standard topological signatures of spaces, such as loops and holes (Gotts, 1994), but also more subtle qualitative features, such as branching points, linear sections, and dead ends. These qualitative features produce fundamental differences in spatial reasoning required for navigating the corresponding environments. For example, the junction point on the $\mathrm{W}$-tracks, which are often used in behavioral experiments (Figure 9C), forces an animal to choose between a right or left turn, which is reflected in the place cell code (Frank et al., 2000; Huang et al., 2009). The RCC5 theory allows capturing such features, e.g., distinguishing between the $U$ - and $W$-tracks, 

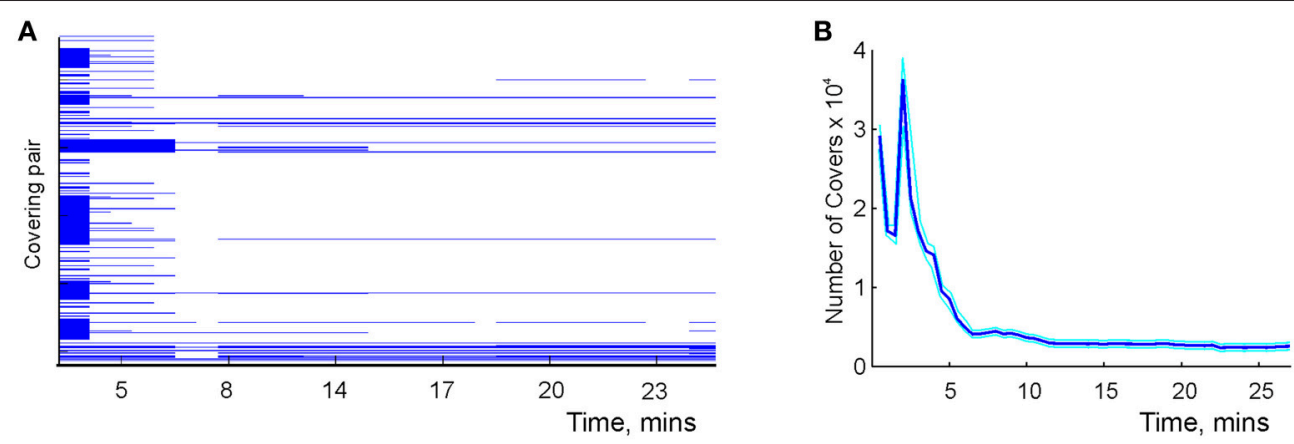

FIGURE 8 | Learning the mereological schema. (A) Time development of the covering relationships: a pair of covering place cells, $U=\left\{c_{i}, c_{j}\right\}$, and a covered cell, $V=\left\{d_{k}\right\}$. Each line corresponds to a specific choice of $U$ and $V$. Each line begins as soon as the covering relationship is detected and stops as soon as it is violated, that is, as soon as the readout neuron shown in Figure 7D would fire. Note that the majority of relationships are short-lived: a large number of spurious relationships are detected at the beginning of exploration. After about $7 \mathrm{~min}$ the majority of them disappear, similar to the behavior of topological loops computed in the simplicial schema shown in Figure 6B. This diagram shows about $1 \%$ of the detected pairs, selected at random. (B) The number of detected cover relationships between pairs of place cells and a single place cell as a function of time.

\section{A}

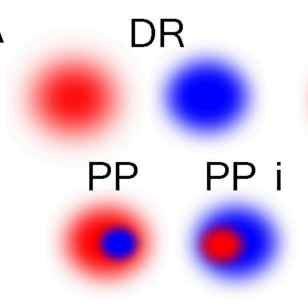

B

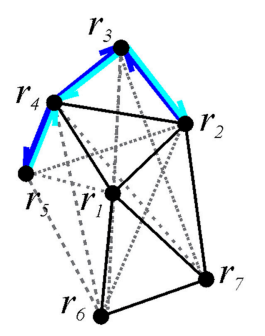

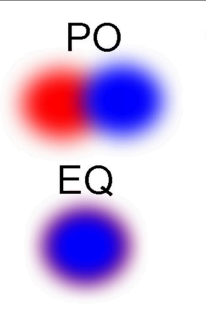
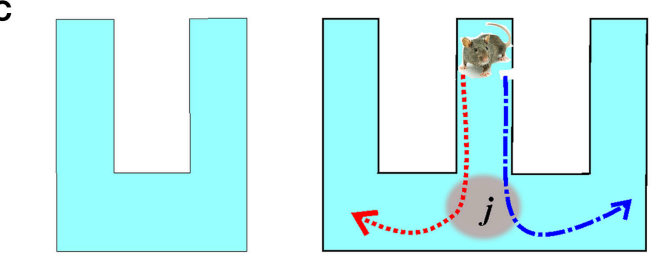

D
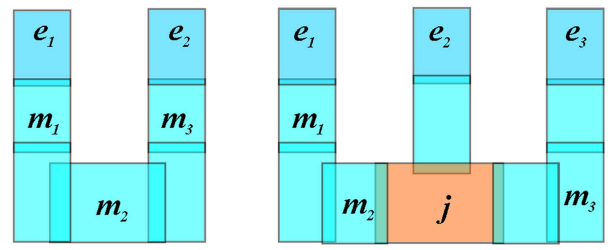

FIGURE 9 | Illustration of RCC5 schema. (A) RCC5 relationships: five logically possible pairwise relations: " $x$ is discrete from $y$ " (denoted as DR), " $x$ partially overlaps with $y$ " (PO), " $x$ is a proper part of $y$ " (PP), " $y$ is a proper part of $x$ " (PPi), and " $x$ is identical to $y$ " (EQ). (B) An RCC5 schema, $\mathcal{R}_{5}$, of the spatial map from Figure 2B. For convenience, the DR connections are shown with gray dashed lines. The structure of the rest of the relations produces a graph similar to the one shown in Figure 2C. The black lines indicate PO connections. Cyan and blue arrows show the PP and PPi connections, respectively. (C) A $U$-track having two dead ends and a $W$-track having three dead ends and a junction, $j$, marked in red. Every time the rat visits the junction point it must choose between the left and the right turn, indicated by the red and blue trajectories, respectively. (D) Topological relationships between regions on a $U$ - and a $W$-track that allow capturing the tracks qualitative geometries. The endpoints, $e_{1}, e_{2}$, and $e_{3}$ are regions that overlap with only one other region. The midpoints, $m_{1}, m_{2}$, and $m_{3}$, overlap with two regions and the junction overlaps with three regions.

which, from the perspective of algebraic topology, are but contractible manifolds (Figure 9D).

To model spatial learning based on a specific RCC approach, one can construct an RCC schema, in which the readout neurons are trained to recognize the appropriate set of binary relationships. However, an important aspect of RCC constructions is that the set of relationships that can be simultaneously imposed on a set of regions is restricted (Bennett, 1998; Renz, 2002). For example, if $x$ and $y$ partially overlap and $y$ is a proper subset of $z$, then $z$ and $x$ must have a non-null intersection and $z$ cannot be a subset of $x$. Therefore, we define an RCC schema $\mathcal{R}$ as a schema with a set of consistent RCC relations between regions.

To model the process of physiological learning in the RCC5 $\left(\mathcal{R}_{5}\right)$ schema, we trained five types of readout neurons to recognize the five RCC5 relationships, starting from the initial DR relationship. This however requires more complex algorithms than in $\mathcal{G}$ and $\mathcal{T}$ schemas: while the partial temporal overlap can always be interpreted as partial spatial overlap, other temporal relationships cannot be uniquely assigned to a spatial RCC5 relation (Figure 10A). For example, passing through two partially overlapping regions along a particular trajectory 
A
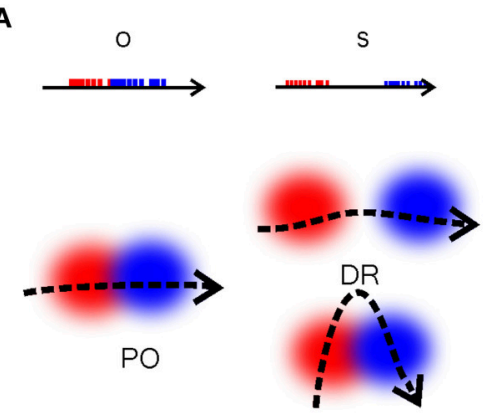

d

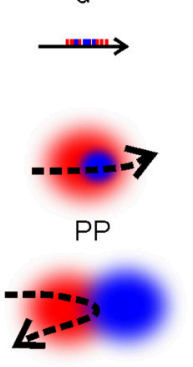

id

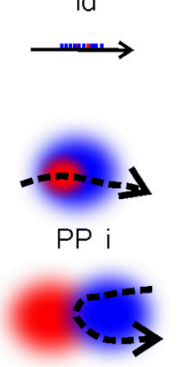

e

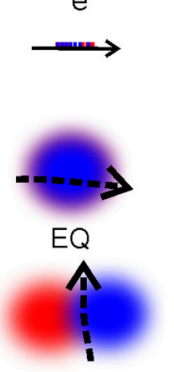

B

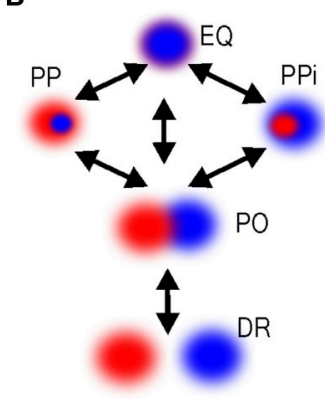

FIGURE 10 | Temporal vs. spatial relationships. (A) Temporal relationships between the spike trains [o, overlap; s, separation; d, during; id, inverse d; and e, equal (Ligozat, 2013)] and the corresponding spatial relationships. The relationships $\mathrm{DR}_{x y}, \mathrm{PP}_{x y}, \mathrm{PPi}_{x y}$, and $\mathrm{EQ}_{x y}$ between the regions can be imitated by partial overlap, depending on the shape of the trajectory, which shows that these relations cannot be directly deduced from the spike train structure. (B) The transitions between the RCC5 relationships, showing the immediate conceptual neighborhood (continuity table) structure of RCC5. These are the possible sequences of gradual transformation of the RCC5 relationships. For example, if at some moment of time two regions, $x$ and $y$, were disconnected (DR $x y$ ) then this relationship cannot instantly jump to a containment relationship $\left(\mathrm{PP}_{x y}\right.$ or $\left.\mathrm{PPi}_{x y}\right)$ without going through, at least instantaneously, the partially overlapping $\left(\mathrm{PO}_{x y}\right)$ relationship.

can generate a temporal disconnect, a temporal cover, or a temporal equality relationship between two spike trains which can be mistaken for spike trains produced by a DR, PP/PPi, or $E Q$ relationship, respectively. Because of this ambiguity, the spiking activity of the presynaptic cells in the cell assemblies produced during individual runs through a pair of place fields can invoke different interpretations of the spatial relationships. Thus, learning a $\mathcal{R}_{5}$ schema rests on encoding, at each moment, the best guesses for the relationships between pairs of regions and then updating them based on the available spiking history and the qualitative analogue of continuity constraints, as shown in Figure 10B.

In our simulations, the relationships evolved rapidly and saturated within about $T_{\mathcal{N}} \approx 4 \mathrm{~min}$ from the onset of the exploration (Figure 11A). Figure 11B shows that at the beginning of the exploration, the number of inconsistencies between independently trained readout neurons is high. Subsequently, their number quickly diminishes as the information about coactivity is acquired. An increase in the number of PP relationships in Figure 11A produces a splash of inconsistencies occurring at about $3 \mathrm{~min}$, which is at the time when the animal completes its first turn around the central hole. This phenomenon has the same origin as the splash of transient cover relationships occurring in the mereological schema $\mathcal{M}$ (Figure 8).

As the statistical information about place cell coactivity accumulates, a stable set of RCC5 relationship emerges. The schema's specific entropy, defined using the probabilities of observing all five relationships, saturates about the same time, $T_{\mathcal{N}} \approx 4 \mathrm{~min}$. The entropy of the RCC5 relationships between the representing regions in the map $M_{X}(\mathcal{R})$ remains similar to the schema entropy during the entire course of learning, reaching the asymptotic value of $H \approx 0.84$ (Figure 11C). Moreover, the mutual information between the map and the schema increases with the acquisition of information in a way similar to the case of graph schema $\mathcal{G}$ but unlike the case of simplicial schema $\mathcal{T}$ (cf. Figures 3C, 5E, 11C). Once again, this data indicates that spatial maps built on regions with diffuse boundaries may better reflect the nature of the encoded regions. In the meantime, the integrals of the $\mathcal{R}_{5}$ schema, i.e., the combinations of RCC5 relationships that represent the junction and the endpoints on the $W$ track, emerge from neuronal spiking in under $T_{\min } \approx 2 \mathrm{~min}-$ much sooner than the readout neurons in $\mathcal{R}_{5}$ network are trained.

\section{DISCUSSION}

We have presented a framework for integrating the place cells' spiking information into a global map of space, implemented via simple cell assembly neural networks, wired to encode spatial relationships. The approach is motivated by the experimental results (Gothard et al., 1996a,b; Shapiro et al., 1997; Poucet and Herrmann, 2001; Lever et al., 2002; Leutgeb et al., 2005; Touretzky et al., 2005; Wills et al., 2005; Alvernhe et al., 2011, 2012; Chen et al., 2012; Dabaghian et al., 2014; Wu and Foster, 2014) and by the theoretical models proposed in Dabaghian et al. (2012); Arai (2014) and in Muller et al. (1996); Trullier and Meyer (2000); Chrastil and Warren (2014). From the perspective of the current approach these models are particular implementations of the simplicial and the graph schema, respectively; the mereological and the RCC schemas are new-to our knowledge, such models have not yet been considered.

In the following we outline several important aspects of this framework and provide a general context for the model.

\subsection{Emergence of the Memory Map in Schemas}

There is a clear parallel between a coherent representation of space emerging from the integrated inputs of many individual neurons and a continuous state of matter (e.g., a solid or a liquid) emerging from the collective dynamics of molecules. From a descriptive point of view, the common element in both phenomena is that neither macro-system can be reduced 
A

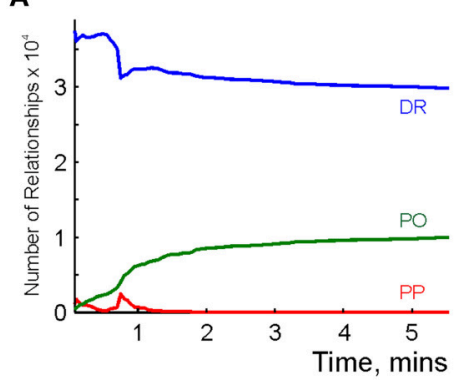

B

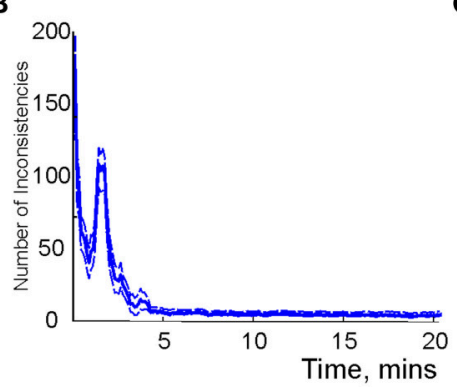

C

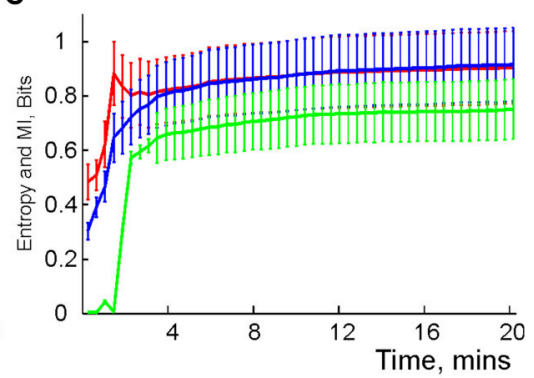

FIGURE 11 | Learning the RCC5 schema. (A) Evolution of RCC5 relationships: at the beginning of the exploration of the new space, the regions are mostly disconnected and the partial overlap of relationships is accumulated over time. Similarly to the graph schema, the number of all types of relationships saturates in about 5 min. (B) The number of inconsistencies in the randomly initialized relational network is higher at the beginning of exploration and decays to a low, steady level by the time the number of relationships stabilize. (C) The entropy of the relations encoded in the schema (red), the entropy of the RCC5 relationships in the map (blue), and the mutual information (green) between them saturate at a similar time scale.

to a trivial aggregation of the properties of its elementary constituents. Even when the properties at both the microscopic and macroscopic levels are well understood, it can be difficult to correlate the properties at one level with those at the other. For example, the measurement of the macroscopic properties of a liquid does not allow one to determine its molecular structure. Conversely, a detailed description of the properties of a water molecule does not explain directly key phenomena of the physics of water. In physics, the solution to this problem historically proceeded from a simplified, phenomenological models, which bridged the gap between the microscopic and macroscopic levels of matter. In a similar way, the present discussion offers a testbed model with which to bridge the gap between place cells and the large-scale spatial map.

\subsection{Topological Spatial Maps}

Topological maps have several biological advantages over geometric [or topographic (Chen et al., 2014)] maps, which follow from the qualitative nature of topological relationships (Chen et al., 2012). First, natural environments are dynamic, so that it is often impossible for an animal to know when and how its navigational task may change. Hence acquiring a qualitative map based on the invariants of the space of an environment, may be biologically more effective than spending time on producing a computationally costly precise answer from mutable relationships between dynamic cues.

One implication of this hypothesis is that in morphing environments the place fields will retain the pattern of topological connectivity and may adjust their shapes in order to compensate for the deformation of the representing space. This hypothesis is supported by experiments which demonstrate that place fields maintain their relative configurations in morphing environments (Muller and Kubie, 1987; Gothard et al., 1996a,b; Lever et al., 2002; Leutgeb et al., 2005; Touretzky et al., 2005; Wills et al., 2005; Colgin et al., 2010) and that place cell coactivity pattern in an animal traversing remains invariant over a significant range of geometric transformations (Diba and Buzsaki, 2008; Dabaghian et al., 2014). If the map is Cartesian, i.e., based on precise coordinates, distances, angles and so forth, such deformation can be achieved by redrawing the place fields at each stage of the deformation, via some complex path integration mechanism (Poucet, 1993; McNaughton et al., 1996; Poucet and Herrmann, 2001; Etienne and Jeffery, 2004; McNaughton et al., 2006; Goodrich-Hunsaker et al., 2008; Alvernhe et al., 2012). From the topological perspective, the observed deformation of the place fields is simply a result of projecting the same stable neuronal map into a morphing environment, which does not require extra computations and hence may be biologically more plausible.

\subsection{Schemas Constrain the Generation of Intrinsic Sequences in the Hippocampus}

Place cells become active in temporal sequences that either match with or are inverse to the spatial ordering of their place fields during the active, resting, or sleep states. Initially, temporal sequences were observed after or during the recording of the place fields, leading various authors to suggest that the observed temporal sequences were a replay of sequences imprinted by sensory inputs (Louie and Wilson, 2001; Foster and Wilson, 2006). More recent experiments have observed temporal sequences that corresponded to trajectories along which the animal had never traveled (Gupta et al., 2010). Furthermore, experiments have revealed that temporal sequences observed before the animal entered an environment for the first time were predictive of the place field sequence measured later (Dragoi and Tonegawa, 2011, 2013). These observations strongly suggest that temporal sequences are not merely replays of previously imprinted sequences (Gupta et al., 2010; Cheng and Ji, 2013). The better interpretation is that sequences are drawn from a pool of sequences that are intrinsic to the hippocampal network and this network structure gives rise to the location of place fields (Buhry et al., 2011; Azizi et al., 2013; Cheng, 2013). The CRISP (for Context Representation, Intrinsic Sequences, and Pattern completion) theory goes further to argue that the intrinsic sequences in the hippocampus are crucial for the storage of episodic memories (Cheng, 2013). However, this theory does not explain the origin or properties of such sequences. 
In the schema framework, all neural activity produced in the hippocampus has to be consistent with its schema. For example, in the graph schema, spontaneously replayed sequences of neural activity would have to be consistent with the connectivity of the graph. In other words, a cell $c_{i}$ may fire a spike after cell $c_{j}$ only if the relationship $\rho_{i j}=\left(r_{i}, r_{j}\right)$, or a chain of intermediate relationships $\rho_{i i_{1}}, \rho_{i_{1} i_{2}}, \ldots, \rho_{i_{n-1} i_{n}=j}$, is present in $P_{R}$. Other schemas impose different constraints on which sequences can be produced, and the elements in the sequences may be ensembles of place cells, rather than single cells. In other words, schemas serve as "topological templates" off which sequences are generated.

Physiologically, this implies that the hippocampal network that implements a particular schema can produce sequences with specific "grammar" which may not have been directly imprinted or previously produced by the network. In fact, such offline state sequences of place cell activations, which the animal had never experienced, were recently observed in the experiments (Dragoi and Tonegawa, 2011, 2013). Moreover, these sequences were consistent with the topology of the spatial environment (Wu and Foster, 2014). Thus, schemas can explain the intrinsic sequences postulated by CRISP theory as well as in preplay and replay. This intimate relationship between spontaneous sequences and schemas may be exploited in future investigations in order to infer the schema based on recordings of sequences or to predict the properties of intrinsic sequences from a given schema.

\subsection{Spatial vs. Non-Spatial Memories}

The hippocampus has been suggested to encode both spatial and nonspatial memories (Eichenbaum et al., 1999; Eichenbaum, 2000; Shrager et al., 2007; Soei et al., 2008; Konkel and Cohen, 2009). For example, it plays a key role in the ability to remember visual, odor, action and memory sequences, and to put a specific memory episode into the context of preceding and succeeding events, as well as the ability to produce complete memory sequence from a single structured input (Wood et al., 1999; Fortin et al., 2002, 2004; Sauvage et al., 2008). The topological view on the hippocampal spatial representations (Dabaghian et al., 2011, 2012, 2014) provides a common framework for understanding both spatial and nonspatial memory functions as manifestations of a single mechanism, which simply produces a topological arrangement of memory elements, irrespective of the nature of their content. According to this view, there is no principal difference between the internalized topological map of spatial locations and a topological map of memory sequences in the mnemonic domain.

\subsection{Connections to Experiment}

Given the place cells' spiking parameters and a hypothesis about how the downstream neurons might process place cell (co)activity, a schematic computation can be used to assess the effectiveness of the corresponding spatial learning mechanism: how much time will be required to map a space, how many integrals can such mapping produce, how quickly these integrals will emerge and how stable they will be. This scope of computations suggests a possibility for experimental verifications of the proposed framework. For example, a decline in spatial learning caused by neurodegenerative diseases (e.g., in
Alzheimer's rat models), by aging or by psychoactive substances is assessed in behavioral experiments in terms of the extra times required to learn various memory tasks. On the other hand, such cognitive changes are associated with changes in the place cell spiking parameters (Gerrard et al., 2001; Silvers et al., 2003; Wilson et al., 2004; Robbe et al., 2006; Cacucci et al., 2008; Robitsek et al., 2008). It may therefore be possible to compare the downturn of spatial memory observed in topological learning tasks (Poucet and Herrmann, 2001; Alvernhe et al., 2012) with the increase of the learning time(s) estimated via a particular schema model for the same change in spiking variables. Another alternative was suggested to us by our recent studies of hippocampal mapping of 3D spaces in bats, using two types of simplicial schemas. The results suggest that in the $3 \mathrm{D}$ case, the readout neurons in the place cell assemblies should operate by integrating synaptic inputs over working memory periods, rather than detecting coactivities on synaptic plasticity timescale (Hoffman et al., 2016). Of course, until these predictions are proved or disproved experimentally, their value is discussable; meanwhile, the schema approach allows theoretical reasoning and generates predictions about hippocampal neurophysiology.

\section{METHODS}

\subsection{Place Cells}

Spiking is produced by the rat's movement through the environment covered by the place fields (Figures 1A,B). The Poisson rate of the firing of place cells is a function of the animal's position $r(t)$ at time $t$,

$$
\lambda_{i}(r)=f_{i} e^{-\frac{\left(r-r_{i}\right)^{2}}{2 s_{i}^{2}}},
$$

where $f_{i}$ is the maximal firing rate of cell $c_{i}, s_{i}$ defines the width of its firing field centered at $r_{i}$ (Barbieri et al., 2004). In an $N$-cell ensemble, the parameters $f_{i}$, and $s_{i}, i=1, \ldots, N$ are modeled as random variables drawn from stationary unimodal distributions characterized by their respective means ( $f$ and $s$ ) and standard deviations (see Figure 1 and Methods in Dabaghian et al., 2012). For the computations we used an ensemble with $N=200$ neurons, with mean firing rate $f=12 \mathrm{~Hz}$ and the mean place field size $s=20 \mathrm{~cm}$. Larger ensembles typically affect the numerical values of the computed quantities, but not the essence of the phenomena described in the paper. This spiking is modulated by theta-oscillations, which are a subcortical EEG cycle in the hippocampus with a frequency of $\sim 8 \mathrm{~Hz}$ (for details see Arai, 2014).

\subsection{Learning Algorithm}

The physiological processes responsible for emergence of cell assemblies with readout neurons trained to integrate presynaptic inputs and to produce a particular response that "actualizes" the information encoded by the place cell coactivity are complex and multifaceted (Buzsaki, 2010). For example, the readout neurons that encode place field overlap must identify a group of place cells and learn to respond to the coactivity of this specific group. However, what matters for our study, are the qualitative results 
of this process: the number of readout neurons, the order of the coactivity detected by these neurons, how this order grows in a typical cell assembly during the learning process and so forth. Therefore, we set aside a neural network simulation of schema learning and employ the following schematic, phenomenological algorithm:

If a relationship $\rho$ of an appropriate type is detected, then:

1. if $\rho$ is already listed in $P_{R}$, ignore;

2. else if $\rho$ can be inferred from the known relationships, ignore;

3. if $\rho$ provides nontrivial information, then add $\rho$ to $P_{R}$.

4. if the known relationships can be inferred from $\rho$, then remove the redundant relationships.

5. continue

Steps 2 and 3 ensure that only the highest order relationships are kept in the schema, eliminating redundant, lower-order relationships. At the beginning, every state of the readout neurons can be empty and trained as the simulated animal explores a novel environment, or these states can be randomly initialized and then relearned. The transitions between the readout neuron types may be regarded as a rudimentary, schematic model of the synaptic plasticity mechanisms. In novel environments, place fields stabilize in about $4 \mathrm{~min}$ (Brown et al., 2001), even though cognitive learning of the environment may take days or even weeks (Frank et al., 2006). This implies that the readout neurons can be trained using constant spiking parameters $f_{i}$ and $s_{i}$.

\subsection{Temporal Relationships}

Temporal Relationships between the spike trains and the physiological mechanisms underlying the downstream neurons' readout process are in general very complex. For the sake of simplicity, we consider only the rate-based representation of neuronal activity (Ahmed and Mehta, 2009), which allows for a variety of possibilities for encoding relationships. Such relationships may entail that the firing rates of the pre- and postsynaptic neurons may be required to fall within a particular interval of values and the period of activity of a neuron $c_{i}$ may be required to precede, to follow, or to overlap with the activity of a neuron $c_{j}$ by a certain minimal, maximal or fixed amount of time (Ligozat, 2013). The present analysis works from the three mutually exclusive logical possibilities for the activity of any two neurons $c_{i}$ and $c_{j}$ :

1. there is an empty intersection of activity, i.e., the two cells are active at different times;

2. there is a non-null intersection of activity, i.e., their activities overlap;

\section{REFERENCES}

Ahmed, O. J., and Mehta, M. R. (2009). The hippocampal rate code: anatomy, physiology and theory. Trends Neurosci. 32, 329-338. doi: 10.1016/j.tins.2009.01.009

Aleksandrov, P. S. (1965). Elementary Concepts of Topology. New York, NY: F. Ungar Pub. Co.

Alvernhe, A., Sargolini, F., and Poucet, B. (2012). Rats build and update topological representations through exploration. Anim. Cogn. 15, 359-368. doi: 10.1007/s10071-011-0460-z
3. the activity of one cell is a proper subset of the other cell, i.e., the activity of one cell occurs entirely within the timespan of the activity of the other cell.

The time window for defining the co-activity of two or more cells is two $\theta$-periods (Mizuseki et al., 2009; Arai, 2014).

Schema entropy and mutual information. For each relationship $\rho_{k}$ of the schema we computed its normalized frequency of appearance $p_{k}$ and evaluated the resulting specific entropy,

$$
H=-\Sigma_{k} p_{k} \log _{2} p_{k}
$$

The specific entropy for the corresponding spatial map was evaluated by identifying the relationships $\rho_{k^{\prime}}$ that obtain between the corresponding representing regions and computing their appearance probabilities $p_{k^{\prime}}$. Following the trajectory of the animal (Figure 1B), we could also detect the joint probability of appearance $p_{k, k^{\prime}}$ of a given pair of relationships, both in the schema as well as in the map $\left(\rho_{k}, \rho_{k^{\prime}}\right)$, and then compute their mutual information between the map and the schema,

$$
M I=-\Sigma_{k} \Sigma_{k^{\prime}} p_{k, k^{\prime}} \log _{2} \frac{p_{k, k^{\prime}}}{p_{k} p_{k^{\prime}}} .
$$

The computational software used for topological analysis is JPlex, an open-source package implementing Persistent Homology Theory methods developed by the Computational Topology group at Stanford University (JPlex freeware, 2011).

\section{AUTHOR CONTRIBUTIONS}

$\mathrm{AB}$ and SC conducted simulations, wrote the paper; YD conceived the idea, conducted simulations, wrote the paper.

\section{ACKNOWLEDGMENTS}

We thank Robert Phenix, Vicky Brandt and Loren Frank for helpful comments. The work was supported in part by Houston Bioinformatics Endowment Fund (AB and YD), the W. M. Keck Foundation grant for pioneering research (AB and $\mathrm{YD})$ and by the NSF 1422438 grant (AB and YD), and by the German Research Foundation (Deutsche Forschungsgemeinschaft, DFG): SFB 874, project B2 (SC), a grant from the Stiftung Mercator (SC).

Alvernhe, A., Save, E., and Poucet, B. (2011). Local remapping of place cell firing in the Tolman detour task. Eur. J. Neurosci. 33, 1696-1705. doi: 10.1111/j.14609568.2011.07653.x

Arai, M., Brandt, V., and Dabaghian, Y. (2014). The effects of theta precession on spatial learning and simplicial complex dynamics in a topological model of the hippocampal spatial map. PLoS Comput. Biol. 10:e1003651. doi: 10.1371/journal.pcbi.1003651

Azizi, A. H., Wiskott, L., and Cheng, S. (2013). A computational model for preplay in the hippocampus. Front. Comput. Neurosci. 7:161. doi: 10.3389/fncom.2013.00161 
Babichev, A., Memoli, F., Ji, D., and Dabaghian, Y. (2015). Combinatorics of place cell coactivity and hippocampal maps. arXiv:1509.01677.

Barbieri, R., Frank, L. M., Nguyen, D. P., Quirk, M. C., Solo, V., Wilson, M. A., et al. (2004). Dynamic analyses of information encoding in neural ensembles. Neural Comput. 16, 277-307. doi: 10.1162/089976604322742038

Barry, C., and Burgess, N. (2007). Learning in a geometric model of place cell firing. Hippocampus 17, 786-800. doi: 10.1002/hipo.20324

Bennett, B. (1998). Determining consistency of topological relations. Constraints 3 , 213-225.

Berge, C. (1982). The Theory of Graphs and Its Applications. Westport, CT: Greenwood Press.

Best, P. J., White, A. M., and Minai, A. (2001). Spatial processing in the brain: the activity of hippocampal place cells. Annual. Rev. Neurosci. 24, 459-486. doi: 10.1146/annurev.neuro.24.1.459

Brette, R. (2012). Computing with neural synchrony. PLoS Comput. Biol. 8:e1002561. doi: 10.1371/journal.pcbi.1002561

Brown, E. N., Frank, L. M., Tang, D., Quirk, M. C., and Wilson, M. A. (1998). A statistical paradigm for neural spike train decoding applied to position prediction from ensemble firing patterns of rat hippocampal place cells. $J$. Neurosci. 18, 7411-7425.

Brown, E. N., Nguyen, D. P., Frank, L. M., Wilson, M. A., and Solo, V. (2001). An analysis of neural receptive field plasticity by point process adaptive filtering. Proc. Natl. Acad. Sci. U.S.A. 98, 12261-12266. doi: 10.1073/pnas.201409398

Buhry, L., Azizi, A., and Cheng, S. (2011). Reactivation, replay, and preplay: how it might all fit together. Neural Plast. 2011:203462. doi: 10.1155/2011/203462

Buzsaki, G. (2010). Neural syntax: cell assemblies, synapsembles, and readers. Neuron 68, 362-385. doi: 10.1016/j.neuron.2010.09.023

Cacucci, F., Yi, M., Wills, T. J., Chapman, P., and O'Keefe, J. (2008). Place cell firing correlates with memory deficits and amyloid plaque burden in Tg2576 Alzheimer mouse model. Proc. Natl. Acad. Sci. U.S.A. 105, 7863-7868. doi: 10.1073/pnas. 0802908105

Chen, A., DeAngelis, G. C., and Angelaki, D. E. (2011). Representation of vestibular and visual cues to self-motion in ventral intraparietal cortex. $J$. Neurosci. 31, 12036-12052. doi: 10.1523/JNEUROSCI.0395-11.2011

Chen, Z., Gomperts, S. N., Yamamoto, J., and Wilson, M. A. (2014). Neural representation of spatial topology in the rodent hippocampus. Neural Comput. 26, 1-39. doi: 10.1162/NECO_a_00538

Chen, Z., Kloosterman, F., Brown, E., and Wilson, M. (2012). Uncovering spatial topology represented by rat hippocampal population neuronal codes. J. Comput. Neurosci. 33, 227-255. doi: 10.1007/s10827-012-0384-x

Cheng, J., and Ji, D. (2013). Rigid firing sequences undermine spatial memory codes in a neurodegenerative mouse model. eLife 2:e00647. doi: 10.7554/eLife.00647

Cheng, S. (2013). The CRISP theory of hippocampal function in episodic memory. Front. Neural Circuits 7:88. doi: 10.3389/fncir.2013.00088

Chrastil, E. R., and Warren, W. H. (2014). From cognitive maps to cognitive graphs. PLoS ONE 9:e112544. doi: 10.1371/journal.pone.0112544

Cohn, A. G., and Hazarika, S. M. (2001). Qualitative spatial representation and reasoning: an overview. Fundam. Inf. 46, 1-29.

Cohn, A. G., Bennett, B., Gooday, J., and Gotts, N. M. (1997). Qualitative spatial representation and reasoning with the region connection calculus. Geoinformatica 1, 275-316.

Colgin, L. L., Leutgeb, S., Jezek, K., Leutgeb, J. K., Moser, E. I., McNaughton, B. L., et al. (2010). Attractor-map versus autoassociation based attractor dynamics in the hippocampal network. J. Neurophys. 104, 35-50. doi: 10.1152/jn.00202.2010

Cui, Z., Cohn, A. G., and Randell, D. A. (1993). "Qualitative and topological relationships in spatial databases," in Proceedings of the Third International Symposium on Advances in Spatial Databases (Singapore: Springer-Verlag), 296-315.

Curto, C., and Itskov, V. (2008). Cell groups reveal structure of stimulus space. PLoS Comput. Biol. 4:e1000205. doi: 10.1371/journal.pcbi.1000205

Dabaghian, Y., Brandt, V. L., and Frank, L. M. (2014). Reconceiving the hippocampal map as a topological template. eLife 3:03476. doi: 10.7554/eLife.03476

Dabaghian, Y., Cohn, A., and Frank, L. (2011). "Topological coding of the hippocampus," in Computational Modeling and Simulation of Intellect: Current State and Future Perspectives, ed B. Igelnik (BMI Research Inc.), 293-320.
Dabaghian, Y., Mmoli, F., Frank, L., and Carlsson, G. (2012). A topological paradigm for hippocampal spatial map formation using persistent homology. PLoS Comput. Biol. 8: e1002581. doi: 10.1371/journal.pcbi.1002581

Dehmer, M., and Mowshowitz, A. (2011). A history of graph entropy measures. Inf. Sci. 181, 57-78. doi: 10.1016/j.ins.2010.08.041

Diba, K., and Buzsaki, G. (2008). Hippocampal network dynamics constrain the time lag between pyramidal cells across modified environments. J. Neurosci. 28, 13448-13456. doi: 10.1523/JNEUROSCI.3824-08.2008

Dragoi, G., and Tonegawa, S. (2011). Preplay of future place cell sequences by hippocampal cellular assemblies. Nature 469, 397-401. doi: 10.1038/nature09633

Dragoi, G., and Tonegawa, S. (2013). Distinct preplay of multiple novel spatial experiences in the rat. Proc. Natl. Acad. Sci. U.S.A. 110, 9100-9105. doi: 10.1073/pnas.1306031110

Dubrovin, B. A., Fomenko, A. T., and Novikov, S. P. (1992). Modern GeometryMethods and Applications. New York, NY: Springer-Verlag.

Eichenbaum, H., Dudchenko, P., Wood, E., Shapiro, M., and Tanila, H. (1999). The hippocampus, memory, and place cells: is it spatial memory or a memory space? Neuron 23, 209-226.

Eichenbaum, H. (1999). Conscious awareness, memory and the hippocampus. Nat. Neurosci. 2, 775-776.

Eichenbaum, H. (2000). A cortical-hippocampal system for declarative memory. Nat. Rev. Neurosci. 1, 41-50. doi: 10.1038/35036213

Etienne, A. S., and Jeffery, K. J. (2004). Path integration in mammals. Hippocampus 14, 180-192. doi: 10.1002/hipo.10173

Fenton, A. A., and Muller, R. U. (1998). Place cell discharge is extremely variable during individual passes of the rat through the firing field. Proc. Natl. Acad. Sci. U.S.A. 95, 3182-3187.

Finkelstein, A., Derdikman, D., Rubin, A., Foerster, J. N., Las, L., and Ulanovsky, N. (2015). Three-dimensional head-direction coding in the bat brain. Nature 517, 159-164. doi: 10.1038/nature14031

Fortin, N. J., Agster, K. L., and Eichenbaum, H. B. (2002). Critical role of the hippocampus in memory for sequences of events. Nat. Neurosci. 5, 458-462. doi: $10.1038 / \mathrm{nn} 834$

Fortin, N. J., Wright, S. P., and Eichenbaum, H. (2004). Recollection-like memory retrieval in rats is dependent on the hippocampus. Nature 431, 188-191. doi: 10.1038 /nature02853

Foster, D. J., and Wilson, M. A. (2006). Reverse replay of behavioural sequences in hippocampal place cells during the awake state. Nature 440, 680-683. doi: 10.1038/nature04587

Frank, L. M., Brown, E. N., and Stanley, G. B. (2006). Hippocampal and cortical place cell plasticity: implications for episodic memory. Hippocampus 16, 775784. doi: 10.1002/hipo.20200

Frank, L. M., Brown, E. N., and Wilson, M. (2000). Trajectory encoding in the hippocampus and entorhinal cortex. Neuron 27, 169-178. doi: 10.1016/S08966273(00)00018-0

Frank, L. M., Stanley, G. B., and Brown, E. N. (2004). Hippocampal plasticity across multiple days of exposure to novel environments. J. Neurosci. 24, 7681-7689. doi: 10.1523/JNEUROSCI.1958-04.2004

Gerevini, A., and Renz, J. (1998). "Combining topological and qualitative size constraints for spatial reasoning," in Proceedings of the 4th International Conference on Principles and Practice of Constraint Programming (Pisa: Springer-Verlag), 220-234.

Gerrard, J. L., Kudrimoti, H., McNaughton, B. L., and Barnes, C. A. (2001). Reactivation of hippocampal ensemble activity patterns in the aging rat. Behav. Neurosci. 115, 1180-1192. doi: 10.1037/0735-7044.115.6.1180

Ghrist, R. (2008). Barcodes: the persistent topology of data. Bull. Am. Math. Soc. 45, 61-75. doi: 10.1090/S0273-0979-07-01191-3

Goodrich-Hunsaker, N. J., Howard, B. P., Hunsaker, M. R., and Kesner, R. P. (2008). Human topological task adapted for rats: Spatial information processes of the parietal cortex. Neurobiol. Learn Mem. 90, 389-394. doi: 10.1016/j.nlm.2008.05.002

Gothard, K. M., Skaggs, W. E., and McNaughton, B. L. (1996a). Dynamics of mismatch correction in the hippocampal ensemble code for space: interaction between path integration and environmental cues. J. Neurosci. 16, 8027-8040.

Gothard, K. M., Skaggs, W. E., Moore, K. M., and McNaughton, B. L. (1996b). Binding of hippocampal CA1 neural activity to multiple reference frames in a landmark-based navigation task. J. Neurosci. 16, 823-835. 
Gotts, N. M., Gooday, J. M., and Cohn, A. G. (1996). A connection based approach to common-sense topological description and reasoning. Monist 79, 51-75.

Gotts, N. (1994). "Defining a 'doughnut' made difficult," in Topological Foundations of Cognitive Science, eds C. Eschenbach, C. Habel, and B. Smith (Hamburg: University of Hamburg), 105-130.

Guger, C., Gener, T., Pennartz, C. M. A., Brotons-Mas, J. R., Edlinger, G., Bermúdez i Badia, S., et al. (2011). Real-time position reconstruction with hippocampal place cells. Front. Neurosci. 5:85. doi: 10.3389/fnins.2011.00085

Gupta, A. S., van der Meer, M. A. A., Touretzky, D. S., and Redish, A. D. (2010). Hippocampal replay is not a simple function of experience. Neuron 65, 695-705. doi: 10.1016/j.neuron.2010.01.034

Harnad, S. (1994). Why and how we are not zombies. J. Conscious. Stud. 1, 164-167.

Harris, K. D., Csicsvari, J., Hirase, H., Dragoi, G., and Buzsaki, G. (2003). Organization of cell assemblies in the hippocampus. Nature 424, 552-556. doi: 10.1038/nature01834

Harris, K. D. (2005). Neural signatures of cell assembly organization. Nat. Rev. Neurosci. 6, 399-407. doi: 10.1038/nrn1669

Hatcher, A. (2002). Algebraic Topology. Cambridge; New York, NY: Cambridge University Press.

Hayman, R., Verriotis, M. A., Jovalekic, A., Fenton, A. A., and Jeffery, K. J. (2011). Anisotropic encoding of three-dimensional space by place cells and grid cells. Nat. Neurosci. 14, 1182-1188. doi: 10.1038/nn.2892

Hazarika, S. M., and Cohn, A. G. (2001). "Qualitative spatio-temporal continuity," in Proceedings of the International Conference on Spatial Information Theory: Foundations of GIS (New York, NY: Springer-Verlag), 92-107. doi: 10.1007/3540-45424-17

Hoffman, K., Babichev, A., and Dabaghian, Y. (2016). Topological mapping of space in bat hippocampus. arXiv:1601.04253

Huang, Y., Brandon, M. P., Griffin, A. L., Hasselmo, M. E., and Eden, U. T. (2009). Decoding movement trajectories through a T-maze using point process filters applied to place field data from rat hippocampal region CA1. Neural Comput. 21, 3305-3334. doi: 10.1162/neco.2009.10$08-893$

JPlex freeware (2011). Computational Topology group, Stanford University. Available online at: http://comptop.stanford.edu/u/programs/jplex

Katz, Y., Kath, W. L., Spruston, N., and Hasselmo, M. E. (2007). Coincidence detection of place and temporal context in a network model of spiking hippocampal neurons. PLoS Comput. Biol. 3:e234. doi: 10.1371/journal.pcbi.0030234

Knauff, M., Rauh, R., and Renz, J. (1997). "A cognitive assessment of topological spatial relations: results from an empirical investigation," Proceedings of the International Conference on Spatial Information Theory: A Theoretical Basis for GIS (Laurel Highlands, PA: Springer-Verlag), 193-206.

Konkel, A., and Cohen, N. J. (2009). Relational memory and the hippocampus: representations and methods. Front. Neurosci. 3:2. doi: 10.3389/neuro.01.023.2009

Lehmann, F., and Cohn, A. G. (1994). "The EGG/YOLK reliability hierarchy: semantic data integration using sorts with prototypes," in Proceedings of the Third International Conference on Information and Knowledge Management (Gaithersburg, MD: ACM), 272-279.

Leutgeb, J. K., Leutgeb, S., Treves, A., Meyer, R., Barnes, C. A., McNaughton, B. L., et al. (2005). Progressive transformation of hippocampal neuronal representations in "morphed" environments. Neuron 48, 345-358. doi: 10.1016/j.neuron.2005.09.007

Lever, C., Wills, T., Cacucci, F., Burgess, N., and O'Keefe, J. (2002). Long-term plasticity in hippocampal place-cell representation of environmental geometry. Nature 416, 90-94. doi:10.1038/416090a

Li, S., and Cohn, A. G. (2012). Reasoning with topological and directional spatial information. Comput. Intell. 28, 579-616. doi: 10.1111/j.14678640.2012.00431.x

Ligozat, G. (2013). Allen's Calculus. Qualitative Spatial and Temporal Reasoning. Hoboken, NJ: John Wiley \& Sons, Inc.

Liu, Y.-M., and Luo, M.-k. (1997). Fuzzy Topology, River Edge, NJ: World Scientific Pub.

Louie, K., and Wilson, M. A. (2001). Temporally structured replay of awake hippocampal ensemble activity during rapid eye movement sleep. Neuron 29, 145-156. doi: 10.1016/S0896-6273(01)00186-6
Maurer, A. P., Cowen, S. L., Burke, S. N., Barnes, C. A., and McNaughton, B. L. (2006). Organization of hippocampal cell assemblies based on theta phase precession. Hippocampus 16, 785-794. doi: 10.1002/hipo.20202

McNaughton, B. L., Barnes, C. A., Gerrard, J. L., Gothard, K., Jung, M. W., Knierim, J. J., et al. (1996). Deciphering the hippocampal polyglot: the hippocampus as a path integration system. J. Exp. Biol. 199, 173-185.

McNaughton, B. L., Battaglia, F. P., Jensen, O., Moser, E. I., and Moser, M. B. (2006). Path integration and the neural basis of the 'cognitive map'. Nat. Rev. Neurosci. 7, 663-678. doi: 10.1038/nrn1932

Mizuseki, K., Sirota, A., Pastalkova, E., and Buzsaki, G. (2009). Theta oscillations provide temporal windows for local circuit computation in the entorhinal-hippocampal loop. Neuron 64, 267-280. doi: 10.1016/j.neuron.2009. 08.037

Mowshowitz, A. (1968). Entropy and the complexity of graphs: I. An index of the relative complexity of a graph. Bull. Math. Biophys. 30, 175-204.

Muller, R. U., and Kubie, J. L. (1987). The effects of changes in the environment on the spatial firing of hippocampal complex-spike cells. J. Neurosci. 7, 1951-1968.

Muller, R., Kubie, J., and Ranck, J. (1987). Spatial firing patterns of hippocampal complex-spike cells in a fixed environment. J. Neurosci. 7, 1935-1950.

Muller, R. U., Stead, M., and Pach, J. (1996). The hippocampus as a cognitive graph. J. Gen. Physiol. 107, 663-694.

O'Keefe, J., and Burgess, N. (1996). Geometric determinants of the place fields of hippocampal neurons. Nature 381, 425-428.

O'Keefe, J., and Nadel, L. (1978). The Hippocampus as a Cognitive Map. New York, NY: Clarendon Press; Oxford University Press.

Poucet, B., and Herrmann, T. (2001). Exploratory patterns of rats on a complex maze provide evidence for topological coding. Behav. Process. 53, 155-162. doi: 10.1016/S0376-6357(00)00151-0

Poucet, B. (1993). Spatial cognitive maps in animals: new hypotheses on their structure and neural mechanisms. Psychol. Rev. 100, 163-182.

Pouget, A., Dayan, P., and Zemel, R. (2000). Information processing with population codes. Nat. Rev. Neurosci. 1, 125-132. doi: 10.1038/35039062

Redish, A. D., and Touretzky, D. S. (1997). Cognitive maps beyond the hippocampus. Hippocampus 7, 15-35.

Renz, J., Rauh, R., and Knauff, M. (2000). "Towards cognitive adequacy of topological spatial relations," in Spatial Cognition II, eds C. Freksa, C. Habel, W. Brauer, and K. Wender (Berlin; Heidelberg: Springer), 184-197.

Renz, J. (2002). Qualitative Spatial Reasoning with Topological Information. Berlin; New York, NY: Springer.

Robbe, D., Montgomery, S. M., Thome, A., Rueda-Orozco, P. E., McNaughton, B. L., and Buzsaki, G. (2006). Cannabinoids reveal importance of spike timing coordination in hippocampal function. Nat. Neurosci. 9, 1526-1533. doi: 10.1038/nn1801

Robitsek, R. J., Fortin, N. J., Koh, M. T., Gallagher, M., and Eichenbaum, H. (2008), Cognitive aging: a common decline of episodic recollection and spatial memory in rats. J. Neurosci. 28, 8945-8954. doi: 10.1523/JNEUROSCI.1893-08.2008

Russell, B. (1921). The Analysis of Mind. London; New York, NY: G. Allen \& Unwin ltd.; The Macmillan company.

Sauvage, M. M., Fortin, N. J., Owens, C. B., Yonelinas, A. P., and Eichenbaum, H. (2008). Recognition memory: opposite effects of hippocampal damage on recollection and familiarity. Nat. Neurosci. 11, 16-18. doi: 10.1038/nn2016

Shapiro, M. L., Tanila, H., and Eichenbaum, H. (1997). Cues that hippocampal place cells encode: dynamic and hierarchical representation of local and distal stimuli. Hippocampus 7, 624-642.

Shrager, Y., Bayley, P. J., Bontempi, B., Hopkins, R. O., and Squire, L. R. (2007). Spatial memory and the human hippocampus. Proc. Natl. Acad. Sci. U.S.A. 104, 2961-2966. doi: 10.1073/pnas.0611233104

Silvers, J. M., Tokunaga, S., Berry, R. B., White, A. M., and Matthews, D. B. (2003). Impairments in spatial learning and memory: ethanol, allopregnanolone, and the hippocampus. Brain. Res. Rev. 43, 275-284. doi: 10.1016/j.brainresrev.2003.09.002

Soei, E., Koch, B., Schwarz, M., and Daum, I. (2008). Involvement of the human thalamus in relational and non-relational memory. Eur. J. Neurosci. 28, 2533-2541. doi: 10.1111/j.1460-9568.2008. 06536.x

Stella, F., Cerasti, E., and Treves, A. (2013). Unveiling the metric structure of internal representations of space. Front. Neural Circuits 7:81. doi: 10.3389/fncir.2013.00081 
Swindale, N. V. (1996). Visual cortex: looking into a Klein bottle. Curr. Biol. 6, 776-779.

Taube, J. S. (2011). Head direction cell firing properties and behavioural performance in 3-D space. J. Physiol. 589, 835-841. doi: 10.1113/jphysiol.2010.194266

Tolman, E. C. (1948). Cognitive maps in rats and men. Psychol. Rev. 55, 189-208.

Touretzky, D. S., Weisman, W. E., Fuhs, M. C., Skaggs, W. E., Fenton, A. A., and Muller, R. U. (2005). Deforming the hippocampal map. Hippocampus 15, 41-55. doi: 10.1002/hipo.20029

Trullier, O., and Meyer, J.-A. (2000). Animat navigation using a cognitive graph. Biol. Cybern. 83, 271-285. doi: 10.1007/s004220000170

Wallgrn, J. O. (2010). Qualitative spatial reasoning for topological map learning. Spat. Cogn. Comput. 10, 207-246. doi: 10.1080/138758609035 40906

Wills, T. J., Lever, C., Cacucci, F., Burgess, N., and O'Keefe, J. (2005). Attractor dynamics in the hippocampal representation of the local environment. Science 308, 873-876. doi: $10.1126 /$ science. 1108905

Wilson, I. A., Ikonen, S., Gureviciene, I., McMahan, R. W., Gallagher, M., Eichenbaum, H., et al. (2004). Cognitive aging and the hippocampus: how old rats represent new environments. J. Neurosci. 24, 3870-3878. doi: 10.1523/JNEUROSCI.5205-03.2004

Wood, E. R., Dudchenko, P. A., and Eichenbaum, H. (1999). The global record of memory in hippocampal neuronal activity. Nature 397, 613-616.
Wu, X., and Foster, D. J. (2014). Hippocampal replay captures the unique topological structure of a novel environment. J. Neurosci. 34, 6459-6469. doi: 10.1523/JNEUROSCI.3414-13.2014

Yartsev, M. M., and Ulanovsky, N. (2013). Representation of three-dimensional space in the hippocampus of flying bats. Science 340, 367-372. doi: $10.1126 /$ science. 1235338

Zeithamova, D., Schlichting, M. L., and Preston, A. R. (2012). The hippocampus and inferential reasoning: building memories to navigate future decisions. Front. Hum Neurosci. 6:70. doi: 10.3389/fnhum.2012. 00070

Zomorodian, A., and Carlsson, G. (2005). Computing persistent homology. Discrete Comput. Geometry 33, 249-274. doi: 10.1007/s00454-004-1146-y

Conflict of Interest Statement: The authors declare that the research was conducted in the absence of any commercial or financial relationships that could be construed as a potential conflict of interest.

Copyright (๑) 2016 Babichev, Cheng and Dabaghian. This is an open-access article distributed under the terms of the Creative Commons Attribution License (CC BY). The use, distribution or reproduction in other forums is permitted, provided the original author(s) or licensor are credited and that the original publication in this journal is cited, in accordance with accepted academic practice. No use, distribution or reproduction is permitted which does not comply with these terms. 